\title{
Improving Time to Palliative Care Review With Predictive Modeling: Study Protocol for a Stepped- wedge, Pragmatic Randomized Controlled Trial
}

Patrick Wilson ( $\nabla$ wilson.patrick@mayo.edu )

Mayo Clinic Minnesota https://orcid.org/0000-0002-6604-444X

Lindsey Philpot

Mayo Clinic Minnesota

Priya Ramar

Mayo Clinic Minnesota

Curtis Storlie

Mayo Clinic Minnesota

Jacob Strand

Mayo Clinic Minnesota

Alisha Morgan

Mayo Clinic Minnesota

Shusaku Asai

Mayo Clinic Minnesota

Jon Ebbert

Mayo Clinic Minnesota

Vitaly Herasevich

Mayo Clinic Minnesota

Jalal Soleimani

Mayo Clinic Minnesota

Brian Pickering

Mayo Clinic Minnesota

\section{Study protocol}

Keywords: Palliative care, electronic medical record, artificial intelligence, Al, pragmatic clinical trials, stepped wedge trials

Posted Date: March 25th, 2021

DOI: https://doi.org/10.21203/rs.3.rs-55430/v1 
License: (c) (i) This work is licensed under a Creative Commons Attribution 4.0 International License. Read Full License 


\section{Abstract}

Background: Palliative care is a medical specialty centered on improving the quality of life (QOL) of patients with complex or life-threatening illnesses. The need for Palliative care is increasing and with that the rigorous testing of triage tools that can be used quickly and reliably to identify patients that may benefit from palliative care.

Methods: To that Aim we will conduct a two-armed stepped-wedge cluster randomized trial rolled out to two inpatient hospitals to evaluate whether a machine learning algorithm accurately identifies patients who may benefit from a comprehensive review by a palliative care specialist, and decreases time to receiving a palliative care consult in hospital. This is a single-center study which will be conducted from August 2019 to November 2020 at Saint Mary's Hospital \& Methodist Hospital, Mayo Clinic, Rochester, Minnesota. Clusters will be nursing units which will be chosen to be a mix of complex patients from Cardiology, Critical Care and Oncology and had previously established relationships with palliative medicine. The stepped wedge design will have 12 units allocated to a design matrix of 5 treatment wedges. Each wedge will last 75 days resulting in a study period of 12 months unless otherwise specified. Data will be analyzed with Bayesian hierarchical models with credible intervals denoting statistical significance.

Discussion: This intervention offers a pragmatic approach to delivering specialty palliative care to hospital patients in need using machine learning, thereby leading to high value care and improved outcomes. It is not enough for Al to be utilized by simply publishing research showing predictive performance; Clinical trials demonstrating better outcomes are critically needed. Furthermore, the deployment of an $\mathrm{Al}$ algorithm is a complex process that requires multiple teams with varying skill sets. To evaluate a deployed $\mathrm{Al}$, a pragmatic clinical trial can accommodate the difficulties of clinical practice whilst retaining scientific rigor.

Trial registration NCT03976297 (ClinicalTrials.gov), registered on June 6, 2019 prior to trial start.

\section{Background}

Palliative care is a medical specialty centered on improving the quality of life (QOL) of patients with complex or life-threatening illnesses through the prevention and management of pain and other problems, be it physical, psychosocial and/or spiritual [1].

As patient populations in western countries are aging and becoming more complex, often requiring care from multiple specialties, there is a growing recognition of the need for specialty palliative care teams to engage with patients earlier in their disease course. A consequence of this increased demand is exemplified by the growing mismatch between clinical care and patient preferences at the end-of-life. For example, research has demonstrated that most people prefer to die at home despite the majority dying outside of the home (nursing home or hospital) [2-3]. Palliative care specialists excel in goals of care and patient preference discussions. Unfortunately, palliative care expertise is often an underutilized 
resource, and the current model of care and incentives may relegate palliative care as a "last resort" after all attempts at cure have been exhausted [4]. This delay can lead to suboptimal symptom management for pain, lower quality of life, and even reduced survival [5-8]. In addition to these unrealized benefits, palliative care has been shown to be inversely associated with aggressive medical interventions and emergency room (ER) visits, hospital and intensive care unit (ICU) admission and death in the last weeks of life, all of which are indicative of low value care [9-11]. Demand for appropriate palliative care will and should increase, and with that policy initiatives and referral triage tools that lead to timely high-quality palliative care services will need to be implemented [12]. Triage tools can be used quickly and reliably to identify patients that may benefit from palliative care, thereby leading to high value care and improved outcomes.

\section{Risk Scores}

The development of risk stratification and triage tools for palliative care services has an extensive history with early methods such as The Palliative Prognostic Score (PaP) $[13,14]$. Although a complete enumeration of scores in this space is out of scope, there are three aspects of palliative care scoring that are important to mention. First, many palliative care tools often use proxies for palliative care need. For example, mortality tends to be the most common proxy usually in the form of the Surprise Question "Would I be surprised if this patient died in the next 12 months?" Although straightforward, mortality as a proxy is substandard, because many patients who are at risk of mortality do not necessarily need palliative care and many patients can benefit from palliative care interaction, even if their risk of short term mortality is low. Other scores have modeled proxies such as use of hospice services but still limit care to end of life and not the entire spectrum of palliative care needs $[15,16]$. Second, palliative care tools often have a targeted timeframe or specified population. For instance, early palliative care scores were focused on patients with cancer, whether late stage or early stage [17]. Subsequently, scores have been developed specifically for inpatient, either general floor or ICU units or community based [18-20]. Although these scores have greater utility, they limit the ability to transfer to other populations which may limit legitimate palliative care need. Third, the complexity of a score is often an important consideration. Earlier models have utilized scores that were simple and easy to calculate, and could be done through clinical examination. Recently with the advent of artificial intelligence (Al) and near universal adoption of electronic medical records (EMR), scores are now being developed that utilizes large and complex types of routinely collected data for predictions. Avati et al. developed a deep learning algorithm on EMR data utilizing a model with 13,654 features predicting mortality, and Jung et al. developed an outpatient mortality prediction algorithm utilizing 1,880 features and using gradient boosting machines (GBMs) [20, 21].

\section{Development of Integrated Palliative Risk Score Algorithm}

Informed by these considerations, a machine learning algorithm trained on EMR data and fully integrated into an IT solution focusing on the identification of patients who may benefit from early palliative care review was developed on inpatients from a large academic medical center. The solution, known as 
Control Tower, pulls disparate data sources centered on a machine learning algorithm which predicts the need for palliative care in hospital. The algorithm along with other key patient indicators was integrated into a graphical user interface (GUI) which allows a human operator, known as the Control Tower Operator (СTO) to review the algorithm predictions and subsequently record the operator's assessment.

\section{Evaluation of Al}

Despite the large number of published risk scores in the literature for palliative care needs, to the best of our knowledge, there has been a lack of research assessing wide scale evaluation and implementation of palliative risk scores into clinical practice. Often scores are tested based solely on predictive performance with validation consisting of clinical review. As hypothesized by Spiegelhalter, it is not enough for algorithms to be trusted by simply publishing research showing predictive performance (Phase I) or clinical assessment (Phase II); two additional phases are needed. First, there should be field testing with clinical trials demonstrating an impact on clinical outcomes (Phase III) as well as an infrastructure to ensure prospective monitoring during routine use [22]. There have been some encouraging and notable exceptions nonetheless; In Courtright et al. the authors published an EHR algorithm for palliative care with a pre-post evaluation on frequencies of consults, advance care planning (ACP) documentation, home palliative care and hospice referrals, code status changes, as well as length of stay [23].

\section{Aims of the Study}

The objective of this study is to evaluate whether the Control Tower solution accurately identifies patients who may benefit from a comprehensive review by a palliative care specialist, and decreases time to receiving a palliative care consult in hospital. By creating an algorithm that automatically screens and monitors patient health status during their hospitalization, the hypothesis being tested is that patients will receive needed palliative care earlier than under the usual course of care.

\section{Trial Design}

To accomplish the primary aim we will use a two-armed stepped-wedge cluster randomized trial rolled out to both inpatient hospitals in Mayo Clinic, Rochester. Trial units will receive a computerized assessment of patients in the hospital identified to be at risk of needing palliative care services where control units will receive the usual source of care.

\section{Methods/design Study Setting}

This is a single-center study which will be conducted from August 2019 to November 2020 at Saint Mary's Hospital \& Methodist Hospital, Mayo Clinic, Rochester, Minnesota. The nursing units chosen for the study have a mix of complex patients from Cardiology, Critical Care and Oncology and had previously established relationships with palliative medicine. 


\section{Eligibility Criteria}

The recruitment and enrollment is broad and is designed to mimic the actual use of the Control Tower in practice. To be included in the trial, patients will need to be admitted to either inpatient facility during the study period. Patients will need to have a risk score of at least 7 (out of 100) from the algorithm. Patients will be excluded from review if they under the age of 18 , previously seen by palliative care during the current hospital encounter, currently enrolled in Hospice or currently followed by a palliative care team. In addition, patients with an expected discharge in the next 24 hours and patients who do not provide research authorization to review their medical records for general research studies in accordance with Minnesota Statute 144.335 will be excluded from the study.

\section{Intervention}

Interventions: A full description of the Control Tower interface can be learned through Murphee et al. [24]. Briefly, the Control Tower is a workstation and software tool that extracts medical data, processes the prediction algorithm, and presents the results through an ordered patient list. Currently the algorithm is running on all inpatients in both study hospitals in an automated monitored process. A screenshot of the interface can be seen in Fig. 1. In addition to the algorithmic score, additional data on comorbidities, laboratory values and hospital events are available and presented to give the score context. Patients receive scores from the Control Tower (0-100; higher score indicating increased need) for palliative care and are subsequently ranked from highest to lowest need with each score colored into tertiles: Red (7 or greater), yellow (less than 7, greater than or equal to 4), and white (less than 4). Patients with previous palliative care in their hospital stay have their scores labeled green.

The Intervention will include a CTO who will interact with the inpatient palliative care consult service at both study sites. The CTO will monitor the Control Tower during weekday normal business hours (Monday through Friday; $8 \mathrm{am}-5 \mathrm{pm}$ ) and select once a day, a cohort of patients with the highest need who may benefit from palliative care review. The operator will assess for any additional exclusion criteria in developing the final list. After all screening is finished the CTO will select the top 12 patients to be sent to palliative care in a file through email. The number 12 was agreed upon to match the expected capacity of the palliative care team and to maintain regularity throughout the trial. The file will consist of the patient identifying information, along with the algorithmic score indicating probability of needing palliative care, and contextual factors such as the hospital unit they are in and what factors in the model are driving the score. The palliative care team member who is on service will also assess the need for each patient through the daily report and record whether they agreed with the algorithm's conclusion or not. For those agreed upon patients who are also in the intervention arm, the palliative care team will approach the attending clinical team to suggest a palliative care referral for the patient.

The utilization of a new tool such as the Control Tower to identify patients appropriate for palliative care can be disruptive to standard clinical workflows and processes. To help ensure proper dissemination of these new referral patterns, we engaged with a communications specialist and worked directly with area practice leaders to set up a communication plan. 
Patients who are not in an intervention period will receive the standard of care. This is feasible given we can easily control the communication between the palliative care team and the attending teams to prevent any contamination between clusters. Furthermore as stated above, we calibrated the prediction model and the Control Tower review to match the average capacity of the palliative care service, knowing that that they will still receive palliative care consults through the traditional pathway i.e. the attending care team consulting palliative care directly. This additional measure increases the likelihood the control group gets the usual source of care.

\section{Outcomes}

For all study outcomes, data will be collected through either the electronic medical record or administrative billing system at trial completion. The primary outcome will be timely identification for need of palliative care as measured by the electronic record of a consult by the palliative care team in the inpatient setting. Data will go through quality checking quarterly, at each step during the burn in period, and before finalization.

The secondary outcomes are as follows:

1. The number of inpatient palliative care consults - Measured by the rate of palliative care consults in the inpatient units of interest.

2. Timely identification for need of palliative care per unit - Measured as time in hours from admission to the electronic record of consult by the palliative care team in the inpatient setting for each of the 12 nursing units.

3. Transition time to hospice-designated bed - For all patients with Medicare insurance the time from admission until transferred to a hospice-designated bed.

4. Time to hospice designation - Measured as time in hours from admission to the electronic record of consult by the hospice care team in the inpatient setting.

5. Emergency Department visit within 30 days of discharge - Measured by the number of study participants who upon discharge from the inpatient setting are readmitted to the Emergency Department at any Mayo Clinic facility within 30 days (excluding inpatient readmissions through the Emergency Department).

6. Hospitalization or readmission within 30 days of discharge - Measured by the number of study participants who upon discharge from the inpatient setting are readmitted to an inpatient unit at any Mayo Clinic facility within 30 days (excluding transfers and planned readmits).

7. ICU transfers - Measured by the number of study participants who transferred to an ICU during their inpatient stay.

8. Ratio of inpatient hospice death to non-hospice hospital deaths - Measured by the number of deaths of study participants in hospice designated beds by the number of deaths in non-hospice beds.

9. Rate of discharge to external hospice - Measured by the number of participants whose electronic health record indicates discharge to external hospice. 
10. Inpatient length of stay - Measured as time from admission to discharge from hospital for all study participants.

\section{Participant timeline}

In the stepped-wedge design clusters, in this case floor units, cross over randomly (computer generated) from the control or standard of care condition to the intervention condition in a staggered fashion. The stepped wedge design will have 12 units allocated to a design matrix of 5 treatment wedges. Each wedge will last 75 days resulting in a study period of 12 months unless otherwise specified. The first step will be a baseline period in which no intervention is administered; where in the last step all clusters will be treated [Table 1)]. At the start of each wedge there will be two weeks of burn-in to allow the clinical team to integrate the intervention with their workflow. Due to the pragmatic nature of the design we are unable to blind providers to whether they were in the intervention unit or control unit.

\section{Data Analysis Plan}

Summarized patient data will be characterized by age, sex, and baseline covariates entered into the machine learning algorithm. All patients will be analyzed on an intention to treat status; this principle will be extended to the cluster status in the event of transfers between intervention and control units.

For all study outcomes Bayesian estimation to account for design features in the stepped design will be used. Specially, time-to-event modeling to assess the effects of the intervention will be used to model timely palliative care and other time to event or count outcomes. The chosen model consists of a hierarchical regression treating the time-to-event as a heterogeneous Poisson process, allowing for adjustment to the event rate due to secular time effects and unit clustering. Unit clustering will be treated with normal random effects and the secular trend will be modeled with autoregressive prior of order 1 . Statistical tests will be based on $95 \%$ credible intervals. For secondary binary outcomes logistic regression will be used with the same design features.

Stepped-wedge cluster randomization trials typically have more statistical power than other cluster randomized designs when clusters are correlated, because each cluster is able to serve as its own control. Because of the complex nature of the design, we estimated statistical power using Monte Carlo simulation [25]. Our model for the simulation consisted of a hierarchical Poisson regression with the outcome being time to palliative care. Random effects for cluster as well as a time series autoregressive model for secular trend were integrated to correctly specify the wedge design. To estimate reasonable parameters for this model we collected pilot data for all Mayo Rochester inpatient admissions in 2017 with palliative care consult status. With estimates of the intra-correlation of clusters, and secular trend estimated from data we have at least $80 \%$ power in several scenarios for the 12 month timeframe to detect Incident rate ratios (IRRs) of 1.25 or greater. See Fig. 1 for the power curves; we tested various scenarios with varying number of clusters and time windows. We choose the 12 month time frame and opted for scenario 2 because it is a good compromise between the power of the test (assuring we could detect a reasonable effect) and implementation (palliative care wanting to make sure they could set up a 
well-defined and accepted process on each of the selected intervention units to ensure intervention fidelity).

\section{Data Management}

All data for all study outcomes, model covariates and process measures will be collected through three principal means:

1. All input predictors and model predictions from the machine learning model are logged every time the algorithm is called and our stored in a study database.

2. Study outcomes will be collected through electronically pulling administrative billing data or data from the health systems EHR.

3. Process measures (number of palliative care accepts and reasons for rejection) will be collected through the daily logs transferred between the СTO and Palliative care team

For each variable pulled electronically we will do a validation study to make sure that it is measuring the appropriate concept.

\section{Data Monitoring}

The proposed intervention does not exceed the threshold of minimal risk so no data monitoring committee (DMC) will be created. Pursuant to this there will be no interim analyses or stopping rules for ending the trial early. Risks of this study to patients are expected to not differ from those encountered during routine clinical care. Patient safety will be maintained through the clinical staff adhering to the standards of clinical care. Study logs will be audited on a quarterly basis for reporting purposes but no statistical analyses will be done and there will be no decisions made on the data to stop or continue the trial.

\section{Confidentiality}

Patient's participation is only through the utilization of hospital services with no additional contact or visits needed; therefore the hospital's policies and procedures for maintaining patient privacy with respect to data will be in place. All patient data are securely stored behind an electronic firewall and will be stored on separate, password-protected, secure servers; only study personnel will have access to these data. For report purposes we will use the Centers for Medicare and Medicaid Services (CMS) data protocol; all results will be reported in aggregate with no cells size smaller than 10 .

\section{Dissemination policy}

Every attempt will be made to have our work published in the literature regardless of outcome and trial summary results will be submitted to ClinicalTrials.gov following the completion of the trial. The team will follow any standard authorship requirements as specified in journals we attempt to publish in.

A checklist of recommended items to address in a clinical trial protocol according to the "Standard Protocol Items: Recommendations for Interventional Trials (SPIRIT) 2013" guidelines is also provided [see 


\section{Discussion}

This study investigates the effect of a machine learning algorithm integrated into a healthcare delivery model to bring timely palliative care to patients in the hospital.

Significant knowledge gaps exist about how risk algorithms routinely perform in practice settings due to the complex nature of the task. Building an algorithm, creating the infrastructure to deliver it in real time, and developing a healthcare model that integrates the algorithm into healthcare delivery and improves outcomes require a large interdisciplinary team. Not surprisingly, the development of such a team is often difficult to create and maintain. In the current paradigm of scientific funding and recognition it may often be a risky decision to engage in team science vs. research which focuses on smaller scale efforts that lead to more research publications $[26,27]$.

Given the nature of this pragmatic clinical trial there are several strengths and limitations that are worth noting. As this trial is imbedded into the clinical practice, the patient population is well represented with very few exclusion criteria and a waiver for both patient and provider consent. Additionally this trial has considerably less burden, as the number and complexity of study visits, study procedures, and questionnaire burden are non-existent and the assessments carried out by study staff are intrinsic to the healthcare model, meaning that if implemented they would be a part of an intervention and are minimal as to not disrupt the usual source of care.

However pragmatic research is not without its limitations. Principally the trial relies on routinely collected data and exclusively uses EHR data for study outcomes. Take our primary outcome as an instance; the choice of timely palliative care is largely a pragmatic measure due to the routine storage of specialty consults in the EHR. Although seemingly more important measures such as unnecessary utilization or quality of life could be ascertained, we opted to target a measure that had adequate power and could be routinely collected throughout the trial without too much burden to practice. In addition the ability to validate EHR measures is limited; the ability to do small validation studies is feasible for key measures but full adjudication is not possible.

\section{Abbreviations}


Table 1

List of Abbreviations

\begin{tabular}{|ll|}
\hline QOL & Quality of Life \\
\hline ICU & Intensive Care Unit \\
\hline ER & Emergency Room \\
\hline El & Artificial Intelligence \\
\hline PaP & Electronic Medical Record \\
\hline GBM & Gradiativent Boosting Machine \\
\hline GUI & Graphical user interface \\
\hline CTO & Control Tower Operator \\
\hline ACP & Advanced Care Planning \\
\hline IRR & Incidence Rate Ratio \\
\hline CMS & Centers for Medicare and Medicaid Services \\
\hline MB & Mary Brigh Unit of Saint Mary's Hospital \\
\hline El & Eisenberg Unit of Methodist Hospital \\
\hline
\end{tabular}

\section{Declarations}

\section{Ethics approval and Consent to Participate}

The protocol for this study was approved as a minimal risk study by the Mayo Clinic Institutional Review Board (IRB) (No. 19-002315). The IRB waived the requirement to obtain individual patient consent and provider consent due to the pragmatic nature of the design. All protocol amendments will be subjected to IRB approval and will be updated subsequently in the clinical trial registry.

\section{Consent for Publication}

Not applicable

\section{Availability of Data and Materials}

De-identified datasets will be available to qualified investigators through communication of reasonable requests with the PI after primary results manuscripts are accepted.

\section{Competing Interests}

The authors have no conflicts of interest or competing interests to disclose. 


\section{Funding}

This study is funded by the Kern Center for the Science of Health Care Delivery and the Clinical Practice Committee (CPC) at Mayo Clinic. The content is solely the responsibility of the authors and does not necessarily represent the official views of the Kern Center or the CPC.

\section{Author Contributions}

P.M.W, C.B.S., PR, and LP developed the study design and drafted the study protocol. P.M.W, C.B.S., PR, LP, and S.W.A developed the analytic and statistical plan. J.S and A.M. provided contributions to the Palliative medicine literature and B.P, J.S, and V.H provided insight on the clinical informatics. All authors will be actively engaged in the implementation of the study protocol. All authors have read, submitted comments and revisions, and approved the final version of this manuscript.

\section{Acknowledgments}

We thank the Center for Palliative Medicine, the IT analysts who developed and monitored the Control tower, and all the patients who participated in our study.

\section{Trial Status}

The protocol published here is version 1.3 dated May 5, 2020. The study began on Aug 19, 2020. Study recruitment commenced on that day and is expected to continue until November 17, 2020. Three events occurred that required a change to the protocol:

1. A logging error was discovered on $12 / 25 / 2019$ which lead to two units not being included in Control Tower: Mary Brigh (MB) 6 D/E and Eisenberg (EI) 4-4. This required one unit MB 6 D/E to be rerandomized because they were already in the intervention and would not have been able to contribute data in the control arm otherwise. El 4-4 was entered into the trial on 11/25/2019; MB6 D/E was entered into the trial on 12/03/2019.

2. On $3 / 18 / 2020$ the clinical trial was paused due to the COVID-19 pandemic. During this time there were no staff available to continue the trial. This pause was ended on 05/04/2020 and there was one week of burn in to allow staff to reorient to the control tower process.

3. An error was discovered in which the algorithm was not correctly calculating scores due to missing data. This delay was further exacerbated by IT furloughs due to the COVID-19 pandemic. As such we decided to remove data during this period (06/05/2020-07/15/2020) and added an additional month of follow up to the last wedge period.

\section{References}

1. WHO definition of palliative care. World Health Organization website. http://www.who.int/ cancer/palliative/definition/en. 
2. Gomes B., Calanzani N., Gysels M., Hall S., Higginson IJ. Heterogeneity and changes in preferences for dying at home: a systematic review. BMC Palliative Care. 2013; DOI: https://doi.org/10.1186/1472-684X-12-7

3. QuickStats: Percentage Distribution of Deaths, by Place of Death - United States, 2000-2014. MMWR Morb Mortal Wkly Rep 2016;65:357. DOI: http://dx.doi.org/10.15585/mmwr.6513a6

4. Top 10 Things Palliative Care Clinicians Wished Everyone Knew About Palliative Care. Strand, Jacob J, Kamdar Mihir M., Elise Carey C., MD Mayo Clinic Proceedings, Volume 88, Issue 8, 859 - 865

5. Temel, J.S., Greer, J.A., Muzikansky, A. et al. Early palliative care for patients with metastatic nonsmall-cell lung cancer. N Engl J Med. 2010; 363: 733-742

6. Greer, J.A., Pirl, W.F., Jackson, V.A. et al. Effect of early palliative care on chemotherapy use and endof-life care in patients with metastatic non-small-cell lung cancer. J Clin Oncol. 2012; 30: 394-400

7. Hui D, Kim SH, Roquemore J, Dev R, Chisholm G, Bruera E. Impact of timing and setting of palliative care referral on quality of end-of-life care in cancer patients. Cancer. 2014;120(11):1743-1749. doi:10.1002/cncr.28628“

8. Earle CC, Park ER, Lai B, Weeks JC, Ayanian JZ, Block S. Identifying potential indicators of the quality of end-of-life cancer care from administrative data. J Clin Oncol. 2003;21(6):1133-38. [PubMed]

9. Zhang, B., Wright, A.A., Huskamp, H.A. et al. Health care costs in the last week of life: associations with end-of-life conversations. Arch Intern Med. 2009; 169: 480-488

10. Chi, Stephen1; Buettner, Benjamin1; Ma, Jessica2; Pollard, Katherine3; Muir, Monica4; Kolekar, Charu5; Al-Hammadi, Noor1; Kollef, Marin1; Dans, Maria1 34: EARLY PALLIATIVE CARE CONSULTATION IN THE MEDICAL ICU: A CLUSTER RANDOMIZED CROSSOVER TRIAL, Critical Care Medicine: January 2019 - Volume 47 - Issue 1 - p 17 doi: 10.1097/01.ccm.0000550826.30295.06

11. Earle CC, Landrum MB, Souza JM, Neville BA, Weeks JC, Ayanian JZ. Aggressiveness of cancer care near the end of life: is it a quality-of-care issue? J Clin Oncol. 2008;26(23):3860-66.

12. Cheng WW, Willey J, Palmer JL, Zhang T, Bruera E. Interval between palliative care referral and death among patients treated at a comprehensive cancer center. J Palliat Med. 2005;8:1025-1032.

13. Marco Pirovano, Marco Maltoni, Oriana Nanni, Mauro Marinari, Monica Indelli, Giovanni Zaninetta, Vincenzo Petrella, Sandro Barni, Ernesto Zecca, Emanuela Scarpi, Roberto Labianca, Dino Amadori, Gino Luporini, A New Palliative Prognostic Score: A First Step for the Staging of Terminally III Cancer Patients, Journal of Pain and Symptom Management, Volume 17, Issue 4, 1999, Pages 231-239, ISSN 0885-3924, https://doi.org/10.1016/S0885-3924(98)00145-6.

14. Marco Maltoni, Oriana Nanni, Marco Pirovano, Emanuela Scarpi, Monica Indelli, Cinzia Martini, Massimo Monti, Ermenegildo Arnoldi, Laura Piva, Alberto Ravaioli, Giorgio Cruciani, Roberto Labianca, Dino Amadori, Successful Validation of the Palliative Prognostic Score in Terminally III Cancer Patients, Journal of Pain and Symptom Management, Volume 17, Issue 4, 1999, Pages $240-$ 247,ISSN 0885-3924, https://doi.org/10.1016/S0885-3924(98)00146-8.

15. Romero-Brufau S, Whitford D, Whitford KJ, Manning DM, Huddleston JM. Identifying patients at risk of inhospital death or hospice transfer for early goals of care discussions in a US referral center: the 
HELPS model derived from retrospective data. BMJ Open. 2018;8(1):e015550. Published 2018 Jan 21. doi:10.1136/bmjopen-2016-015550

16. Meffert C, Rücker G, Hatami I, Becker G. Identification of hospital patients in need of palliative care-a predictive score. BMC Palliat Care. 2016;15:21. Published 2016 Feb 22. doi:10.1186/s12904-0160094-7

17. Stacy M. Fischer, Wendolyn S. Gozansky, Angela Sauaia, Sung-Joon Min, Jean S. Kutner, Andrew Kramer, A Practical Tool to Identify Patients Who May Benefit from a Palliative Approach: The CARING Criteria, Journal of Pain and Symptom Management, Volume 31, Issue 4, 2006, Pages 285292, ISSN 0885-3924, https://doi.org/10.1016/j.jpainsymman.2005.08.012.

18. Anderson F, Downing GM, Hill J, Casorso L, Lerch N. Palliative Performance Scale (PPS): a new tool. J Palliat Care. 1996;12:5-11

19. Villa G, De Gaudio AR, Falsini S, Lanini I, Curtis JR. Development of END-of-Life ScorlNG-System to identify critically ill patients after initial critical care who are highly likely to die: a pilot study. Minerva Anestesiol 2015 December;81(12):1318-28.

20. Jung K, Sudat SEK, Kwon N, Stewart WF, Shah NH. Predicting need for advanced illness or palliative care in a primary care population using electronic health record data. J Biomed Inform 2019;92:103115 doi:

21. Avati A, Jung K, Harman S, Downing L, Ng A, Shah NH. Improving palliative care with deep learning. BMC Med Inform Decis Mak 2018;18(Suppl 4):122 doi: 10.1186/s12911-018-0677-8[published Online First: Epub Date].

22. Spiegelhalter, D. (2020). Should We Trust Algorithms? Harvard Data Science Review, 2(1). https://doi.org/10.1162/99608f92.cb91a35a

23. Courtright, K.R., Chivers, C., Becker, M. et al. Electronic Health Record Mortality Prediction Model for Targeted Palliative Care Among Hospitalized Medical Patients: a Pilot Quasi-experimental Study. J GEN INTERN MED 34, 1841-1847 (2019). https://doi.org/10.1007/s11606-019-05169-2

24. Dennis H. Murphree, Patrick M. Wilson, Shusaku W. Asai, Daniel J. Quest, Yaxiong Lin, Piyush Mukherjee, Nirmal Chhugani, Jacob J. Strand, Gabriel Demuth, David Mead, Brian Wright, Andrew Harrison, Jalal Soleimani ,Vitaly Herasevich, Brian W. Pickering, Curtis B. Storlie, (2020). Improving the Delivery of Palliative Care Through Predictive Modeling and Healthcare Informatics. Journal of the American Medical Informatics Association, 2021; ocaa211, https://doi.org/10.1093/jamia/ocaa211

25. Landau, S., \& Stahl, D. (2013). Sample size and power calculations for medical studies by simulation when closed form expressions are not available. Statistical Methods in Medical Research, 22(3), 324-345. https://doi.org/10.1177/0962280212439578

26. Pasterkamp, G., Hoefer, I. \& Prakken, B. Lost in the citation valley. Nat Biotechnol 34, 1016-1018 (2016). https://doi.org/10.1038/nbt.3691

27. Bennett LM, Gadlin H. Collaboration and team science: from theory to practice. J Investig Med. 2012;60(5):768-775. doi:10.2310/JIM.0b013e318250871d 
Figures

\begin{tabular}{|c|c|c|c|c|}
\hline $\begin{array}{l}\text { Patient, Test } \\
57 \text { years I Male } \\
4 \text { days in the hospital }\end{array}$ & $\begin{array}{l}\text { Faciliy: } \\
\text { Dept } \\
\text { Room: }\end{array}$ & $\begin{array}{l}\text { Problems List } \\
\text { 1. Anemia Posthemonthagic Acute (Blood Loss Anemia) } \\
\text { 2. Primary Open.Angle Glaucoma Mild Stage Blateral } \\
\text { 3. Age Reated Nuclear Cataract Buaseral } \\
\text { 4. Uvetis Anterior Acute }\end{array}$ & 54 total - & $\begin{array}{l}\text { 24-hour Events } \\
\text { 1. Nursing Services CARE PLAN } \\
\text { 2 Gastroenterology and Hepasology PROGRESs } \\
\text { 3. Gastroenterology and Hepasclogy Disch Summ } \\
\text { 4. Nursing Services CARE PLAN }\end{array}$ \\
\hline
\end{tabular}

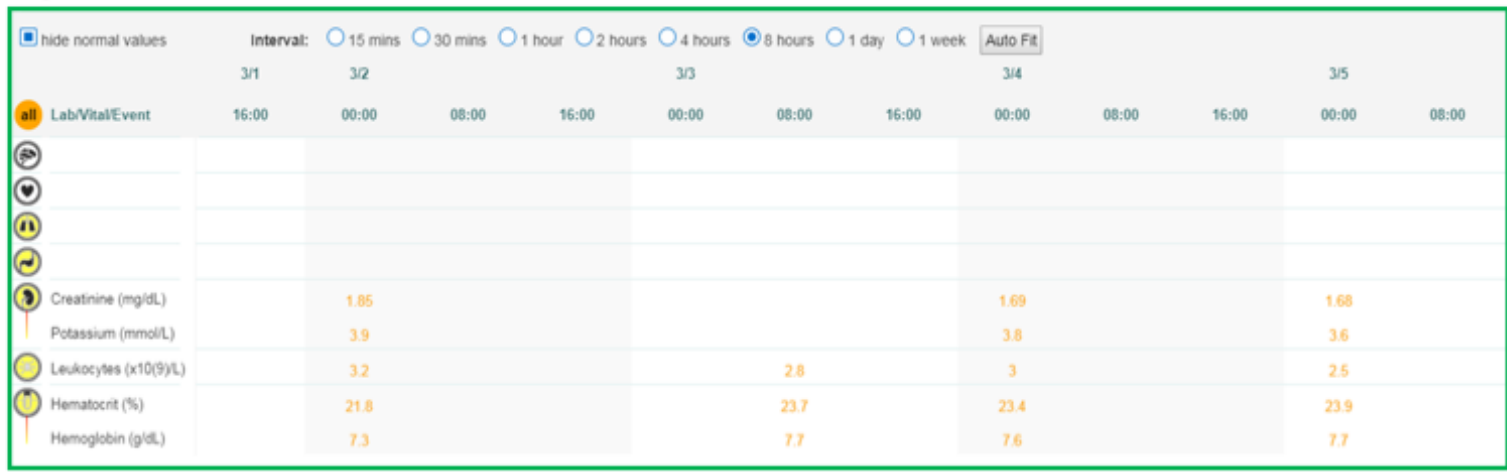

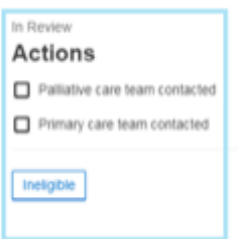

Artificial Intelligence

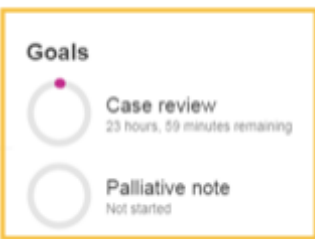

oct sarios

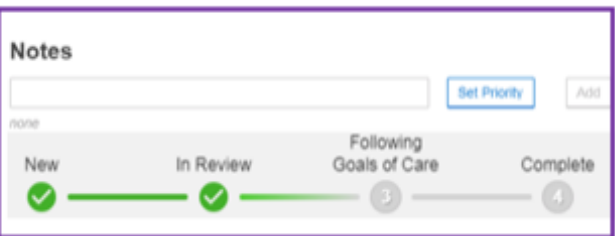

Defined QI Goals
Communication

\section{Figure 1}

Screenshot of the Control Tower User Interface 


\begin{tabular}{|c|c|c|c|c|c|c|c|}
\hline \multirow{2}{*}{$\begin{array}{l}\text { Cluster List } \\
\text { ET } 7\end{array}$} & \multicolumn{2}{|c|}{$\begin{array}{l}\text { Baseline } \\
\text { Aug 19-Oct } \\
31\end{array}$} & $\begin{array}{l}\text { Time } 1 \\
\text { Nov 01-Jan } \\
13\end{array}$ & $\begin{array}{l}\text { Time } 2 \\
\text { Jan 14-May } \\
20\end{array}$ & $\begin{array}{l}\text { Time } 3 \\
\text { May 21-Aug } \\
2\end{array}$ & \multirow[t]{2}{*}{$\begin{array}{l}\text { Time } 4 \\
\text { Aug } 3-\text { Nov } \\
16\end{array}$} & \multirow[t]{2}{*}{$\begin{array}{l}\text { Close } \\
\text { Nov } \\
17 \\
\end{array}$} \\
\hline & & & & \begin{tabular}{l|l}
$x$ & \\
\end{tabular} & \begin{tabular}{|l|l}
$\mathrm{x}$ & \\
\end{tabular} & & \\
\hline EI $10-3 / 10-4$ & & & & $\mathrm{x}$ & $\mathrm{X}$ & & \\
\hline $\mathrm{MB} 5 \mathrm{~B} / \mathrm{G}$ & & & & $x$ & $\mathrm{x}$ & & \\
\hline $\mathrm{MB} 6 \mathrm{D} / \mathrm{E}$ & $\mathrm{X}$ & $X X X X X X$ & $X X$ & $\mathrm{X}$ & $\mathrm{X}$ & & \\
\hline Unit 4-3 & & & & $\mathrm{x}$ & $\mathrm{X}$ & & \\
\hline ET 5 & & & & $\mathrm{x}$ & $\mathrm{X}$ & & \\
\hline ET 4 & & & & $\mathrm{X}$ & $\mathrm{x}$ & & \\
\hline ET 6 & & & & $x$ & $x$ & & \\
\hline El 4-4 & $\mathrm{x}$ & $X X X X X X$ & $X X$ & $x$ & $\mathrm{X}$ & & \\
\hline \multicolumn{8}{|l|}{$\begin{array}{l}\text { Data } \\
\text { Collection }\end{array}$} \\
\hline $\begin{array}{l}\text { Model Log } \\
\text { Data }\end{array}$ & 0 & 0 & 0 & 0 & 0 & 0 & \\
\hline $\begin{array}{l}\text { Process } \\
\text { Measures }\end{array}$ & 0 & 0 & 0 & 0 & 0 & 0 & \\
\hline $\begin{array}{l}\text { Study } \\
\text { Outcomes }\end{array}$ & & & & & & & 0 \\
\hline
\end{tabular}

The trial will be conducted over 12 months with 12 inpatient units crossing from control to intervention in 75 day steps. Each cluster will have a burn-in period of 2 weeks at the start of the trial and when they receive the intervention. White cells denote usual source of care, and grey cells are intervention periods. The Xs signify when the intervention was silent, and $\mathrm{Os}$ indicate period when data collection occurs.

Figure 2

Stepped wedge design 


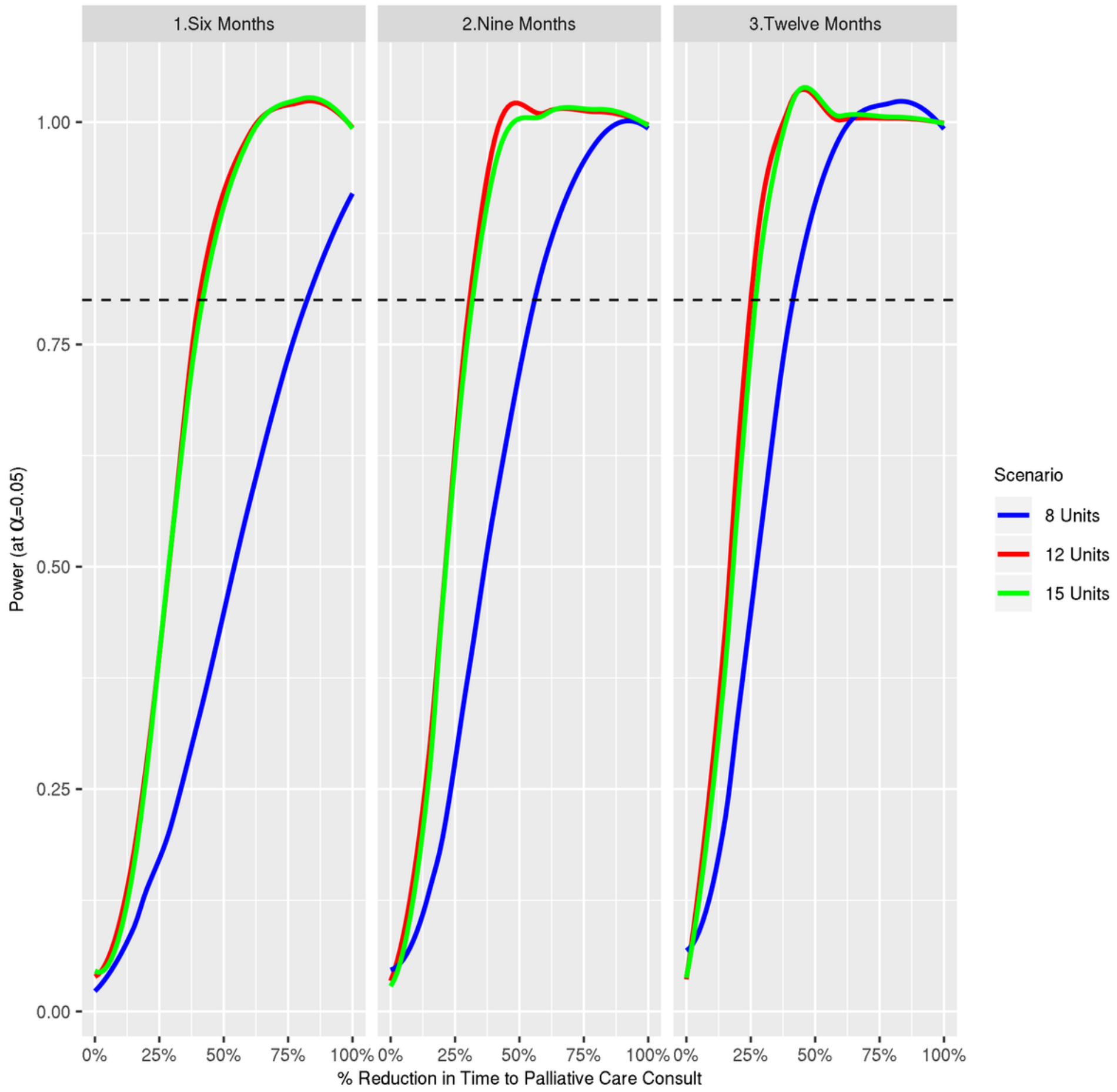

Figure 3

Stepped Wedge Design Power Curves

\section{Supplementary Files}

This is a list of supplementary files associated with this preprint. Click to download. 
- SPIRITCTchecklistv2.doc

Page 18/18 